\title{
CMV incidence in Scottish renal transplant units; a comparison between two different approaches to CMV prophylaxis
}

Kolb AM, Henderson L, Geddes C, Walbaum D, Joss N, Henderson D, Alfonzo A and Petrie MC on behalf of the Scottish Renal Registry.

\section{Introduction}

* Of the two Scottish renal transplant centres, Glasgow gives 6 months of Valganciclovir prophylaxis only to CMV negative recipients of CMV positive transplants (CMV D+/R-), while Edinburgh gives the same dose and duration of Valganciclovir to all except CMV negative recipients of CMV negative transplants (CMV D-/R-).

* Neither unit routinely screens for asymptomatic CMV viraemia.

* The most common side effects of Valganciclovir relate to bone marrow suppression (leucopenia, anaemia and thrombocytopenia).

* Both Edinburgh and Glasgow Transplant units give Basiliximab with methylprednisolone induction and Tacrolimus, Mycophenolate mofetil (MMF) and prednisolone for maintenance immunosuppression.

\section{Methods}

* Study population included all renal transplant recipients transplanted in 2015 in Scotland

* Follow-up data was collected for the Scottish Renal Registry Transplant Outcome Census: donor and recipient characteristics, induction and maintenance immunosuppression, complications (including rejection, infection, malignancy, diabetes mellitus, surgical complications) as well as graft and patient outcomes.

* CMV disease defined as CMV viraemia plus one or more of the following: leucopenia, deranged LFTs, fever/raised inflammatory markers with no alternative explanation, and/or diarrhoea.

* Incidence of leucopenia +/- resultant reduction/cessation of valganciclovir (and/or mycophenolate mofetil) was also examined, allowing comparison between patients receiving valganciclovir, and those who did not.

\section{Results}

* There were 235 kidney transplants into adult recipients in Scotland in 20I5; I 34 in Glasgow and III in Edinburgh.

* Immunosuppression and baseline characteristics of donor and recipients were comparable between units.

* There were fewer CMV D-/R- transplants ( $16 \%$ versus $28 \%, p=0.03)$ and more CMV D+/R+ transplants (33\% versus $21 \%, p=0.007)$ in Edinburgh versus Glasgow.

* Nationally there were 20 cases of CMV viraemia (incidence $8.3 \%$ ) and 10 cases of CMV disease (4.1\%).

* The proportion developing CMV viraemia in each CMV mismatch group differed between Glasgow and Edinburgh transplant units (see figure I), with higher rates of viraemia in the West (I7/I34 versus $3 / \mathrm{II}, \mathrm{p}=0.00 \mathrm{I}$ ) but comparable rates of CMV disease between units $(3 / 134$ versus $7 / 110, p=0.2)$.

* Most (75\%) of Glasgow's CMV cases presented less than 6 months post-transplant compared to $33 \%$ in Edinburgh $(p=0.09)$.

* There were no deaths solely attributable to CMV during the followup period, but one patient died from pneumocystis jirovecii pneumonia whilst being treated for CMV disease.

- Rejection rates were comparable (13\% in Glasgow versus $16 \%$ in Edinburgh, $\mathrm{p}=0.3$ ) at median follow-up 284 days (IQR 202-395 days).

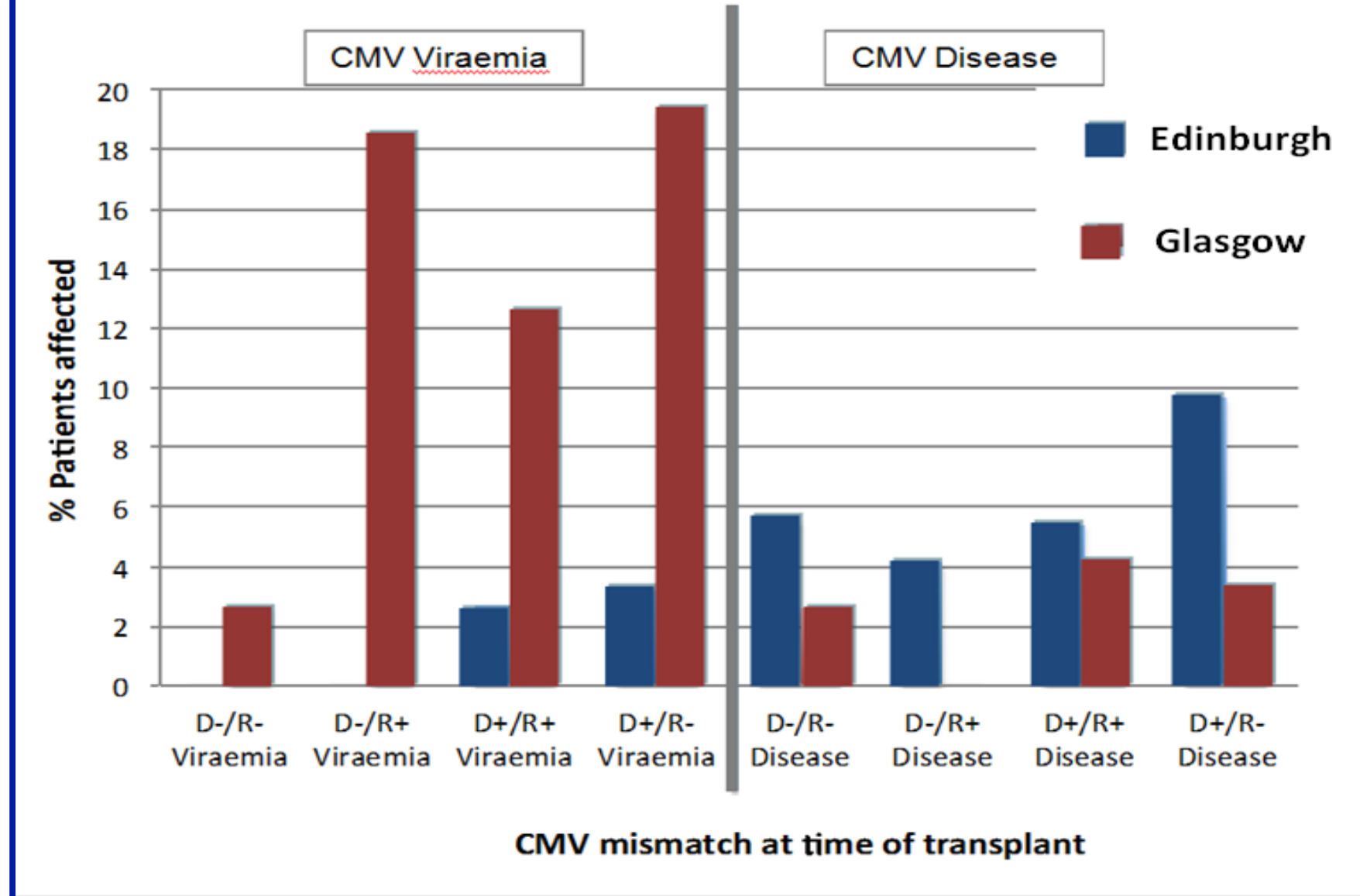

Figure I. Rates of CMV viraemia and CMV disease according to baseline CMV mismatch at the time of transplantation

* 32\% of patients given Valganciclovir did not complete 6 months of prescribed treatment; in all cases leucopenia was the reason for Valganciclovir cessation.

- MMF was reduced as a result of leucopenia in 10\% in Edinburgh versus $9.7 \%$ in Glasgow $(p=0.9)$, despite more widespread use of Valganciclovir in Edinburgh.

\section{Discussion}

* The comparable CMV disease rates and absence of clear benefit in giving more widespread Valganciclovir prophylaxis may favour the Glasgow approach to prophylaxis, in an effort to minimise pill burden and potential side effects of Valganciclovir.

* Similarly, the lack of evidence of harm or adverse effects to suggest Valganciclovir has a negative influence on patient outcome would support Edinburgh's approach.

* It is unclear why there is a trend to higher rate of CMV disease in those transplanted in Edinburgh, despite more widespread prescription of Valganciclovir and further investigation to compare overall immunosuppression burden (e.g. tacrolimus trough levels) between units is required.

* Rates of CMV viraemia will be influenced by local threshold for serological testing or if routine screening is policy; units using less Valganciclovir may conceivably test more often for CMV and therefore appear to have higher (asymptomatic) viraemia rates.

* Local rates of rejection and immunosuppression burden will impact on CMV risk and must be considered when deciding local prophylaxis policy.

* Any change in prophylaxis policy should be followed by close monitoring of CMV incidence, but also rejection and details of immunosuppression to allow full assessment of the relative risks. 